\title{
Distinction of Electromyography Signal in Normal and Neuropathy Patients Using Wavelet Feature
}

\author{
Jun-Woo Lee', Se-Jin Ahn², Myung-Jun Shin³, Myung-Hun Jang³, Weui-Bong Jeong1 \\ ${ }^{1}$ School of Mechanical Engineering, Pusan National University \\ 2, Busandaehak-ro 63beon-gil, Geumjeong-gu, Busan, Republic of Korea \\ qwer5209@pusan.ac.kr; wbjeong@pusan.ac.kr \\ ${ }^{2}$ Division of Energy and Electric Engineering, Uiduk University \\ 261, Donghae-daero, Gangdong-myeon, Gyeungju-si, Gyeongsangbuk-do, Republic of Korea \\ sjahn@uu.ac.kr \\ ${ }^{3}$ Department of Rehabilitation Medicine, Pusan National University Hospital \\ 179, Gudeok-ro, Seo-gu, Busan, Republic of Korea \\ drshinmj@gmail.com; zmh1048@naver.com
}

\section{Extended Abstract}

Electromyography (EMG) is a technique for measuring motor unit action potential (MUAP) occurring in muscle during muscle contraction[1]. Common types of EMG include surface EMG and needle EMG. The surface EMG is a method of measuring the synthesized signal of MUAPs by attaching the patch to the skin. On the other hand, individual MUAP can be measured because needle EMG is a method of invading a needle into a muscle[2]. In patients with neuropathy, the undamaged nerve transmits larger and more complex signals to the muscle fiber to compensate for damaged nerves. Therefore, EMG signals in patients with neuropathy are larger and more complex than those of normal subjects[3]. However, the physical characteristics, the location of the measurement, and the degree of activity of the muscles have a significant effect on the EMG signal measurement. Therefore, in order to effectively analyze the characteristics of the EMG signal generated repeatedly over a very short time, there have been various studies using various signal processing techniques. In this study, wavelet transform (WT) was applied to the needle EMG signal to distinguish between neuropathic patients and normal subjects. WT is a signal processing technique that represents the signal through scaling and position shifting of the wavelet function. Therefore, it has an advantage in analyzing frequency characteristics over time. The time signal is transformed to wavelet coefficients in time-scale domain by WT. Three features calculated by wavelet coefficients showed differences in neuropathy and normal. As a result, three features of normal subjects were within certain ranges, on the other hand those of neuropathic patients were outside those ranges. These features are possible to obtain a more distinct result than using features of existing research. Therefore, features proposed in this study can be helpful to diagnose neuropathic disorders.

\section{Acknowledgements}

This research was supported by the Bio \& Medical Technology Development Program of the NRF funded by the Korean government, MSIP(2018M3A9E8942066)

\section{References}

[1] M. B. Raez, M. S. Hussain, and F. Mohd-Yasin, "Techniques of EMG signal analysis: detection, processing, classification and applications," Biol Proced Online, vol. 8, pp. 11-35, 2006.

[2] G. R. Naik, S. E. Selvan, and H. T. Nguyen, "Single-Channel EMG Classification With Ensemble-Empirical-ModeDecomposition-Based ICA for Diagnosing Neuromuscular Disorders," IEEE Trans Neural Syst Rehabil Eng, vol. 24, no. 7, pp. 734-43, 2016.

[3] P. Artameeyanant, S. Sultornsanee, and K. Chamnongthai, "An EMG-based feature extraction method using a normalized weight vertical visibility algorithm for myopathy and neuropathy detection," Springerplus, vol. 5, no. 1, p. 2101, 2016. 